\title{
THz Reflection Spectroscopy of Explosives Measured by Time Domain Spectroscopy
}

\author{
N. PALKA* \\ Institute of Optoelectronics, Military University of Technology \\ S. Kaliskiego 2, 00-908 Warsaw, Poland
}

\begin{abstract}
Reflection spectra of pure explosive materials and their simulants were measured in transmission and reflection by means of time domain spectroscopy in the range 0.1-3 THz. Spectra measured in two reflection configurations: specular with incident angle equal to $45^{\circ}$ and stand-off with close to normal incidence and distance 30 and $40 \mathrm{~cm}$ to samples was analyzed.
\end{abstract}

PACS: 07.57.Ty, 07.57.Pt, 78.20.Ci, 78.40.Pg

\section{Introduction}

Terahertz range of electromagnetic spectrum $(0.1 \div$ 10) $\mathrm{THz}$ has focused attention of scientists for years because of many interesting features including existence of spectral fingerprints of many materials (also explosives), through clothing imaging and safety for human being. Such advantages are of great importance for biochemical, defense and security related applications.

Scanning of pure explosives in transmission mode is limited to samples with thickness less than about $1 \mathrm{~mm}$, which is connected with relatively high attenuation especially in absorption peaks regions. In case of thicker samples one should consider a reflection way of measurement. We examined $100 \%$ pure samples made of explosives: hexogen (RDX), penthryte (PETN), octogen (HMX), their mixtures (RDX + PETN, RDX $+\mathrm{HMX})$ as well as sugar and simulants of explosives - L-tartaric acid and 4-aminobenzoic acid (PABA). Spectra were acquired using commercial time domain spectroscopy (TDS) setup from TeraView (TPS Spectra 3000) with appropriate accessories in atmosphere with humidity less than $1 \%$. We carried out our experiments using 3 configurations: transmission, specular reflection with incident angle $45^{\circ}$ and stand-off reflection with 30 and $40 \mathrm{~cm}$ distance to a sample.

\section{Sample preparation}

Sample preparation is a very important factor to obtain reliable spectra. If the particle size of a solid material is comparable to the $\mathrm{THz}$ wavelength $(100-500 \mu \mathrm{m})$, the scattering may cause a significant change in spectrum of measured sample [1-3]. The materials were ground using a mortar and pestle to reduce the particle size and to avoid scattering loss. Afterwards, the samples were pressed into pellets directly using a hydraulic press

* e-mail: npalka@wat.edu.pl
(2 tons). For transmission investigations we mixed $10 \%$ of the explosive or the simulant with $90 \%$ of polyethylene (HDPE) to obtain a $400 \mathrm{mg}$ sample with $13 \mathrm{~mm}$ diameter. A pure polyethylene sample (360 mg) was used as a reference. For reflectance analysis, we manufactured pellets which consisted of pure materials with weights of $400 \mathrm{mg}$ and $13 \mathrm{~mm}$ diameter. A gold mirror was used as a reference.

\section{Setup}

Time domain spectroscopy is a commonly used technique in $\mathrm{THz}$ range [1]. Teraview TPS 3000 unit with accessories was used for transmission and reflection investigations. The main parameters of the systems are: spectral range 0.06-3.6 THz, signal-to-noise better than 4000:1, dynamic range optical density higher than 3 in the range $2 \mathrm{~cm}^{-1}$ to $100 \mathrm{~cm}^{-1}$, spectral resolution $0.06 \mathrm{THz}$ and rapid scan mode with 30 scans/second.

For better understanding of interaction between $\mathrm{THz}$ waves and the explosive materials we used standard transmission setup and two reflection configurations specular and stand-off:

1) In specular reflection mode (Fig. 1a), the sample is placed close to the emitter and the detector and the incidence and collection angles of the beam are $45^{\circ}$. The accessory is installed inside the measurement chamber of the spectrometer.

2) Stand-off measurements were carried out in an external free standing module with a fibre-fed emitter and detector (Fig. 1b). In this case, $\mathrm{THz}$ beam by means of a set of mirrors was focused on the sample and after specular reflection was collected and focused on the detector. Samples were measured in the compartment purged with dry air (humidity less than 1\%) in the distances 30 and $40 \mathrm{~cm}$ between a sample and the mirrors. Incident angles were 5 and 4 degree for 30 and $40 \mathrm{~cm}$ distance, respectively. The measurement time was a few minutes depending on averaging and length of the scan required for the analysis. 


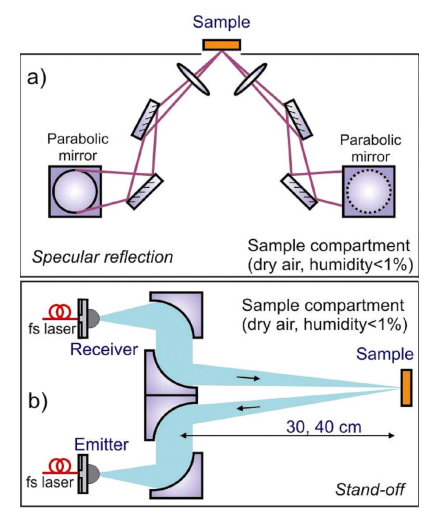

Fig. 1. Specular reflectance accessory (a) and stand-off measurement unit (b).

Absorbance $(A)$ of a sample, which is determined as $-\log _{10}(T)$, where $T$ is transmission of a sample, is commonly used as transmission units. One of the advantages of TDS technique is a possibility to calculate refractive index $(n)$ of a sample using a simple formula, which bases on a phase difference between incident and transmitted spectra $[4,5]$. In the considered case, $\mathrm{THz}$ waves illuminate a flat surface of the sample and therefore the reflected $\mathrm{THz}$ beam with a reflection angle the same as the incident angle can be theoretically treated using the Fresnel equation and reflectance $(R)$ can be approximately calculated by the formula [4]:

$$
R \approx\left(\frac{n-1}{n+1}\right)^{2} .
$$

On the other hand, reflectance can be experimentally determined in reflection configuration setup by division of the measured power spectrum, by a power spectrum from a mirror reference, measured in an otherwise identical fashion [5].

\section{Results and discussion}

Figure 2a presents transmission spectra of RDX and calculated on this basis its refractive index. Reflection spectra of RDX calculated by means of refractive index (Eq. (1)) as well as measured in specular and both stand-off configurations are shown in Fig. 2b. Dashed line represents peak in absorbance of RDX and center of slope of reflectance.

It is clearly seen from Fig. 2a that sharp absorbance peak at $0.84 \mathrm{THz}$ is connected with the fall of the refractive index. Also distinct spectral features in reflectance are present at this frequency. The agreement between calculated and experiment spectra is good. In case of shape of characteristics, the calculated spectrum is similar to the specular reflection spectrum because in this setup the sample is close to the emitter and the detector and is not disturbed by propagation issues. In terms of values, the calculated spectrum is similar to $40 \mathrm{~cm}$ stand-off setup in $0.8 \mathrm{THz}$ range due to the fact that incident angle in this setup is close to normal assumed in Eq. (1).

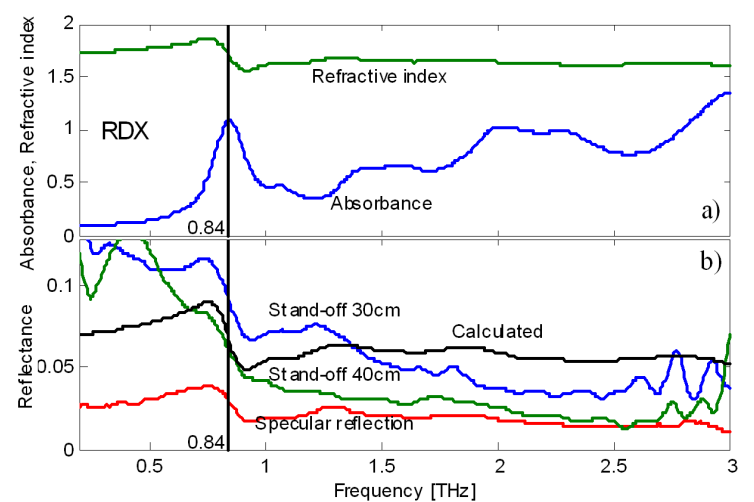

Fig. 2. Octogen (RDX): transmission spectrum and refractive index (a) and calculated and measured reflection spectra (b).

Discrepancies at higher frequencies are connected with low sensitivity of the arrangement and long propagation distance.

Figure 3 shows spectral behavior of tartaric acid both in transmission and reflection (specular and both stand-off) configurations. Basing on Fig. $2 \mathrm{~b}$ and $3 \mathrm{~b}$ one can note that specular characteristics are more flat before and after the main slope. For stand-off configurations the reflectance is more changeable in regions outside the main slope. Moreover, differences between maximum and minimum values of the reflectance slope are bigger for both stand-off configurations in comparison to $45^{\circ}$ specular reflection.

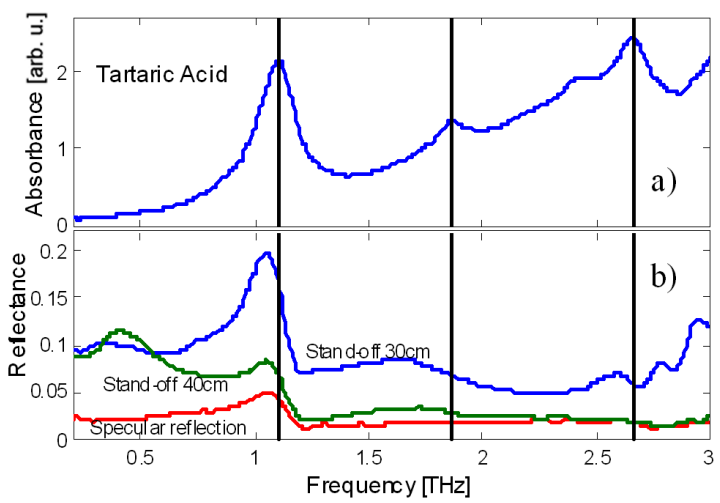

Fig. 3. Tartaric acid: transmission (a) and reflection (b) spectra.

Values of reflectance for specular case are in the range 0.01-0.05 while for stand-off configurations higher values up to 0.2 are observed. For the measured materials, $30 \mathrm{~cm}$ stand-off distance between the sample and the mirrors gives more distinct slopes compared to $40 \mathrm{~cm}$ distance due to the smaller propagation distance.

Figures 4 and 5 present transmission and $45^{\circ}$ specular reflection spectra of PETN and sugar as well as HMX and PABA, respectively. We grouped these materials to show 


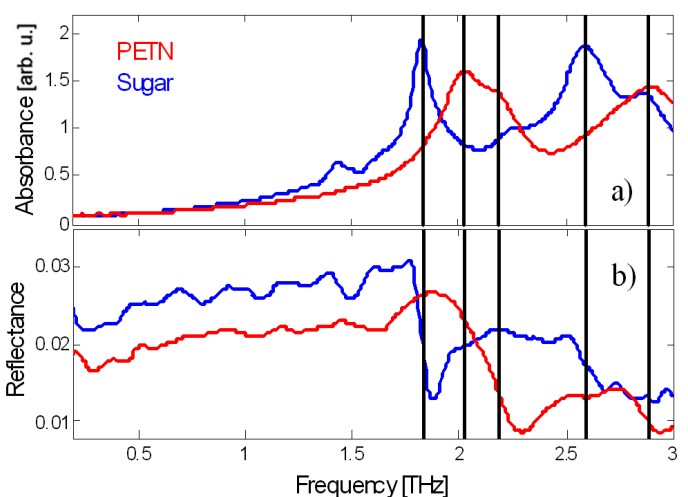

Fig. 4. PETN and tartaric acid - transmission (a) and $45^{\circ}$ specular reflection spectra (b).

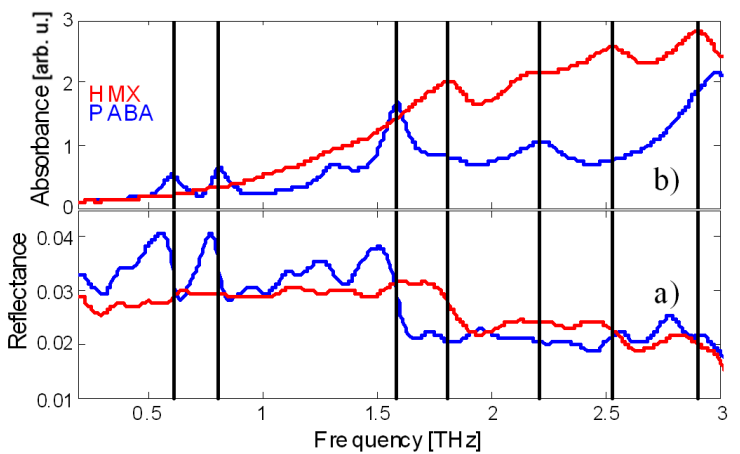

Fig. 5. HMX and PABA - transmission (a) and $45^{\circ}$ specular reflection spectra (b).

that the simulants (sugar and PABA) have characteristic fingerprints in the same frequency range as explosives and can be used in measurements to eliminate the risks associated with the explosion. Dashed and dot lines are connected with absorbance or reflectance features of appropriate substances.

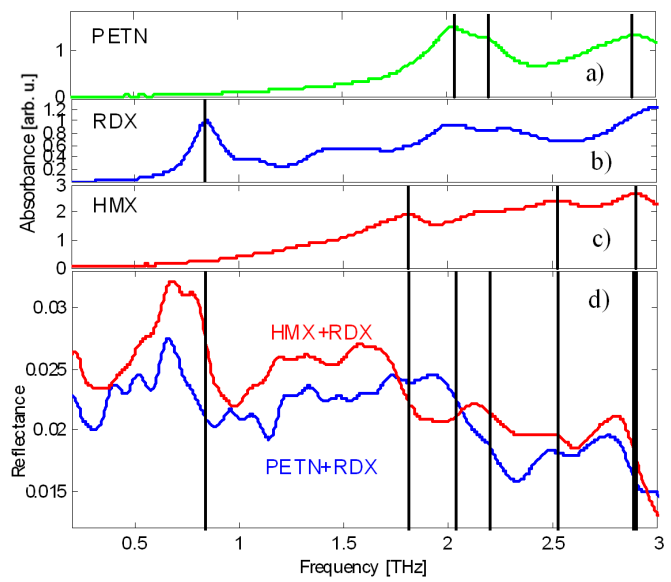

Fig. 6. Transmission spectrum of PETN (a), RDX (b), HMX (c) and reflection spectra of HMX + RDX and PETN +RDX (b).
Transmission spectra of PETN, RDX, and HMX are shown in Fig. 6a-c, respectively, and are similar to other groups results $[4,6]$. Mixtures of explosives can be also found in different applications, e.g. PETN mixed with RDX and other minor additives form a plastic explosive called semtex. Figure 6d presents reflection spectra of two mixtures: HMX and RDX (red) and PETN and RDX (blue). It can be seen that peaks in absorbance of the individual substances (dashed, dot and dashed-dot lines) are associated with drops in reflectance of their mixtures.

It should be also noted that contrast (difference between maximum and minimum) in reflection analysis is significantly less than in transmission measurements, but distinctive features are still observed for frequencies connected with the absorbance peaks. This situation renders stand-off detection of explosives difficult. Moreover, in real applications one should take into account also effects of propagation geometry, clothing barriers and atmospheric absorption which makes detection even more challenging [5].

\section{Summary}

Spectra of pure explosives (RDX, HMX, PETN), their mixtures and simulants (sugar, tartaric acid and PABA) were measured in transmission and reflective modes in TDS system. Two reflection configurations were applied: specular with incident angle equal to $45^{\circ}$ and stand-off with close to normal incidence with 30 and $40 \mathrm{~cm}$ distance to the sample. Peaks in absorbances of all measured substances are associated with features in reflectance. This result reveals that $\mathrm{THz}$ reflection measurement can be used for stand-off detection of explosives but strong technological progress is needed in the field of generation and detection of terahertz radiation.

\section{Acknowledgments}

The project is financed by the Polish Ministry of Science and Education under the project O R00 003412.

\section{References}

[1] N. Palka, Acta Phys. Pol. A 118, 128 (2010).

[2] J. Chen, Y. Chen, H. Zhao, G. Bastiaans, X.-C. Zhang, Opt. Expr. 15, 12060 (2007).

[3] K. Barczak, T. Pustelny, D. Dorosz, J. Dorosz, Europ. Phys. J.-Special Topics 154, 11 (2008).

[4] N. Palka, W. Ciurapinski, J. Wróbel, Acta Phys. Pol. A 116, 368 (2009).

[5] M.C. Kemp, C. Baker, I. Gregory, NATO Security through Science Series, IOS Press Books Online, Brussels 2006, p. 151.

[6] M.R. Leahy-Hoppa, M.J. Fitch, X. Zheng, L.M. Hayden, R. Osiander, Chem. Phys. Lett. 434, 227 (2007). 